Nervenarzt 2012 $\cdot 83: 1363$

DOI 10.1007/s00115-012-3552-3

Online publiziert: 17. Oktober 2012

(c) Springer-Verlag Berlin Heidelberg 2012
P. Falkai

Klinik für Psychiatrie und Psychotherapie, Ludwig-Maximilians-Universität München

\section{Zukunft der} psychosozialen Medizin
Psychosoziale Medizin bezieht sich auf alle psychologischen und soziologischen Aspekte des Krankseins. Diese Faktoren spielen bei der Entstehung, dem Verlauf und der Bewältigung von Krankheiten eine bedeutsame Rolle. Auch für die Förderung und den Erhalt der Gesundheit sind psychosoziale Faktoren essenziell.

Die psychosoziale Medizin verfolgt das Ziel, den Patienten durch eine interdisziplinäre Zusammenarbeit und umfassende Betrachtung unter Berücksichtigung psychosomatischer Aspekte und Einbeziehung somatischer Komorbiditäten möglichst in seiner Gesamtheit zu erfassen. Den individuellen Voraussetzungen und Bedürfnissen entsprechend soll den $\mathrm{Pa}$ tienten eine optimale und effiziente Therapie ermöglicht werden.

In der alltäglichen Arbeit ist die Kommunikation und Interaktion mit dem Patienten und häufig auch den Angehörigen ein wichtiger Faktor. Erst das individuelle Eingehen auf den Patienten, gepaart mit einem Blick über den Tellerrand in andere Disziplinen, ermöglicht die optimale Versorgung des Patienten.

\section{ע) Die psychosoziale Medizin begleitet den Patienten bei seinem psychischen Genesungsprozess}

Die heutige Entwicklung der medizinisch-psychiatrischen Versorgung ist geprägt von demographischen Veränderungen, zunehmender Morbidität und medizinischen Fortschritten. Gleichzeitig sehen wir uns mit knapper werdenden Ressourcen konfrontiert. In Zeiten der ständigen Kostensenkung und Gewinnopti- mierung in der Versorgung psychisch erkrankter Patienten stellt sich die Frage, ob eine solche zuwendungsorientierte Medizin gegenüber der weit verbreiteten kostenorientierten Sichtweise noch Chancen hat. Die eigentliche ärztliche und therapeutische Aufgabe, sich dem Patienten in seiner Individualität, seiner Geschichte und Lebensumwelt zuzuwenden, droht immer mehr in den Hintergrund zu geraten. Das Ziel der psychosozialen Medizin, die Patienten bei ihrem psychischen Genesungsprozess zu begleiten und $\mathrm{zu}$ unterstützen, ist in Gefahr.

Die Akzeptanz psychischer Erkrankungen ist nach wie vor begrenzt. Wir setzen uns ein sowohl für die Anerkennung unserer Arbeit als Fachärzte für Psychiatrie und Psychotherapie als auch für die Anerkennung und bestmögliche Versorgung von Menschen mit psychischen Erkrankungen. Dies gilt im Hinblick auf die Gesundheitspolitik ebenso wie auf die gesellschaftliche Wahrnehmung.

Deshalb machen wir uns auch stark für eine fächerübergreifende Stärkung psychosomatisch-psychosozialer Versorgungsaspekte in der medizinisch-psychiatrischen Behandlung. Hierzu gehört in allererster Linie eine bessere Integration psychiatrischer, psychotherapeutischer und somatisch orientierter Behandlungsansätze.

Was erwartet Sie konkret in diesem Heft?

Das vorliegende Schwerpunktheft zur Zukunft der psychosozialen Therapie kann natürlich nur einige der vielen Teilaspekte dieser Thematik herausgreifen. So beschäftigen sich Mathias Berger et al. zum Thema Burn-out mit geeigneten Interventions- und Versorgungsmaßnahmen. Steffi Riedel-Heller und Kolle- gen beleuchten das Thema Depression im Alter im Hinblick auf demographische Entwicklungen sowie mögliche zukünftige Optimierungswege. Linn Kühl et al. vertiefen das Thema Depression in einer anderen Richtung: Sie beschreiben diese als Risikofaktor für kardiovaskuläre Erkrankungen. In einem weiteren Beitrag beschreibt Karl-Jürgen Bär, wie die Schmerzforschung zum besseren Verständnis psychiatrischer Erkrankungen beitragen kann, und Hans Kurt und Kollegen fassen in ihrem Beitrag die verschiedenartigen Entwicklungen der Psychosomatik in den anderen deutschsprachigen Ländern und Regionen Europas zusammen.

Das Leitthema des diesjährigen DGPPN-Kongresses lautet „Zukunft der Psycho-Sozialen Medizin“. Der Kongresstitel hätte abgeändert genauso gut heißen können „Zukunft: Die Psycho-Soziale Medizin“.

In diesem Sinne wünsche ich Ihnen bei der Lektüre der folgenden Seiten ebenso wie auf unserem Kongress 2012 vielfältige Impulse und rege Diskussionen.

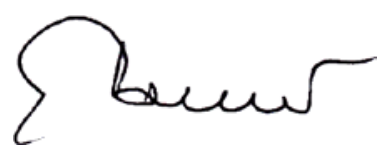

Prof. Dr. P. Falkai

\section{Korrespondenzadresse}

Prof. Dr. P. Falkai

Klinik für Psychiatrie und Psychotherapie, Ludwig-Maximilians-Universität München Nussbaumstr. 7, 80336 München

Peter.Falkai@med.uni-muenchen.de

Interessenkonflikt. Der korrespondierende Autor gibt an, dass kein Interessenkonflikt besteht. 